\title{
The effects of bud load and regulated deficit irrigation on sugar, organic acid, phenolic compounds and antioxidant activity of Razakı table grape berries
}

\author{
Semih Tangolar ${ }^{1}$, Güzin Tarım¹ ${ }^{1}$ Haşim Kelebek ${ }^{2}$, Serpil Gök Tangolar ${ }^{1}$, and Sevilay Topçu ${ }^{3}$ \\ ${ }^{1}$ Department of Horticulture, Faculty of Agriculture, Çukurova University, Adana, Turkey \\ ${ }^{2}$ Department of Food Technology, Faculty of Engineering and Natural Sciences, Adana Science and Technology \\ University, Adana, Turkey \\ ${ }^{3}$ Department of Agricultural Structures and Irrigation, Faculty of Agriculture, Çukurova University, Adana, Turkey
}

\begin{abstract}
This study aims at assessing the effects of increased bud load and irrigation applications on berry quality of the Razakı table grape. Two Regulated Deficit Irrigation (RDI) having different irrigation levels (RDI-I and RDI-II) based on the growth stages, in addition to a non-irrigated control treatment together with two different bud load practices (K-normal and 2K-two-fold buds of the normal) were examined for their effects on quality attributes such as sugar and organic acids contents, phenolic compounds as well as antioxidant capacity of the berries. The non-irrigated vines had highest sugar level $(198.86 \mathrm{~g} / \mathrm{kg})$ in the first year (2013) of the experiment whilst the sugar content of the berries was increased with irrigation (RDI-II) in 2014. However the highest organic acid $(7.10 \mathrm{~g} / \mathrm{kg}$ ) was recorded from the RDI-II treatment in 2013 whereas those of from non-irrigated vines were highest $(7.81 \mathrm{~g} / \mathrm{kg})$ in 2014 . Considering the sugar and organic acid content of the berries, bud load effects were not significant. The total phenolic acids were higher under non-irrigated and $2 \mathrm{~K}$ bud load conditions. Antioxidant activity of berries was increased with RDI-I irrigation and 2K practices in the first year (2013) although no significant effect was recorded in the second year of the experiment. In all applications, glucose among the sugars, tartaric acid among the organic acids, catechin and epicatechin among the phenolic compounds were detected to be higher compared to other components in berries.
\end{abstract}

\section{Introduction}

In places with an annual rainfall of 500-600 mm, non-irrigated viticulture can be performed depending on the soil type. However, a supplementary irrigation might be necessary to maintain high yield and quality of the grapes when the seasonal distribution of the rainfall is not appropriate [1]. Irrigation management as a tool for use in the production of grapes has continued to receive attention in many regions of the world, and the regulated deficit irrigation (RDI) is one of the strategies which have been used intensively in vineyards [2-4]. RDI further aims to allow a certain amount of water deficit on the grapevine especially between berry set and maturation periods [5-7]. In wine grape varieties, RDI may increase berry and wine quality by decreasing vegetative growth [8] and accordingly allowing more sunlight and optimum temperature conditions in microclimate around clusters send by this way it may increase skin/pulp ratio, phenolic compounds, anthocyanins and antecedent aroma compounds [9-11]. The excessive water as well as its shortage may solely affect the photosynthesis activity, shoot elongation, berry development, berry size, color and juice composition, depending on the vine nutritional status and yield charge. Ojeda et al. [12] examined the effects of various levels of deficit irrigation on phenolic compounds of Shiraz grape. Effect was realized by decreasing berry size or directly effecting biosynthesis and could be positive or negative depending on the type of phenolic compound. Besides, many researches [13-15] also stated that the total phenolic compounds, antioxidant capacities and phytochemical properties depend on grape variety, climate, soil conditions, maturation levels, cultural applications and yield amount. In this study, the effect of different RDI and bud load applications on berry quality parameters related with human health such as sugars, organic acids, phenolic compounds and antioxidant capacity $[8,16-19]$ in Razakı grape variety were investigated.

\section{Materials and methods}

This study was carried out in a vineyard with sixteen years old table grape $c v$. Razakı (Vitis vinifera L.) in Pozanti Agricultural Research Center (1080 m alt.) of Çukurova University (Adana, Turkey) in 2013 and 2014. The own rooted vines were cultivated under non-irrigated condition. Vine rows were planted with an east/west orientation using a rectangular configuration of $3 \times 2 \mathrm{~m}$. The training system was bilateral cordon trellised to a two-wire vertical system. The soil in experimental vineyard has slightly alkaline character, with a clay-loam and loam texture on the upper soil and 30-60 cm depth, respectively. Field capacity and the permanent wilting point were $27.16 \mathrm{~cm}^{3} \mathrm{~cm}^{-3}$ and $19.39 \mathrm{~cm}^{3} \mathrm{~cm}^{-3}$, respectively. 
Three irrigation treatments including non-irrigated as the control, and two irrigation treatments (RDI-I and RDI-II) together with two bud load treatments, traditional $(\mathrm{K})$ and twofold bud loads $(2 \mathrm{~K})$, were investigated in the experiment. In both RDI treatments, the irrigation time was scheduled according to the midday leaf water potentials (LWP) while the irrigation amount applied was calculated using the cumulative evaporation (Epan) values obtained from Class-A Evaporation Pan. The midday LWP values used for irrigation timing for both RDI treatments were the same, however these values differed at each plant growth stage: $-1.0 \mathrm{MPa}$ for before flowering period, $-1.3 \mathrm{MPa}$ between berry set-veraison and between veraison-ripening, and $-1.2 \mathrm{MPa}$ for post-ripening stage. Irrigation amounts applied to the RDI-I plots were $50 \%$ and $75 \%$ of the cumulative evaporation from the Class A Pan during the growth stages berry set-veraison and veraison-ripening, respectively, whilst these percentages were $75 \%$ and $50 \%$ of the evaporation from the Pan for the same growth stages, respectively. Two bud loads; control $(\mathrm{K})$ which is traditional practice (20 buds for the first $500 \mathrm{~g}$ of pruning weight, and 10 more buds for every additional $500 \mathrm{~g}$ subsequently) [1,20,21] and increased bud loads (2K-double amount of control) (Table 1) were investigated in all irrigation treatments (non-irrigated, RDI-I and RDI-II). Pruning was done on March 2013 and 2014.

Irrigation water amount was calculated using following equation based on cumulative Class A pan evaporation within the irrigation intervals.

$$
I=A \times E \text { pan } \times \mathrm{Kpc} \times P
$$

I: Irrigation water amount (L), $A$ : parcel area $\left(\mathrm{m}^{2}\right), E$ pan: Evaporation from the class-A pan ( $\mathrm{mm}), \mathrm{Kpc}$ : The crop and pan coefficients (0.6), $P$ : Percentage of wetted area $(50 \%)$.

Irrigation was applied using drip irrigation system and the drip lines were placed close to a vine row. Fertilizers were applied in two occasions, beginning of February

Table 1. Retained bud number ( $n$ ) by pruning weight (kg/vine) in Regulated Deficit Irrigation (RDI) and bud load of Razakı grapevine.

\begin{tabular}{|c|c|c|c|c|c|}
\hline Year & Irrigation & Bud load $^{1}$ & \multicolumn{2}{|c|}{$\begin{array}{c}\text { Pruning } \\
\text { weight }\end{array}$} & $2 K$ \\
\hline 2013 & \multicolumn{2}{|c|}{ All Treatments } & 700 & 24 & 48 \\
\hline \multirow{11}{*}{2014} & \multirow{3}{*}{ No irrigation } & K & 1050 & 31 & 62 \\
\hline & & $2 \mathrm{~K}$ & 1113 & 32 & 64 \\
\hline & & Mean & 1082 & 31 & 62 \\
\hline & \multirow{3}{*}{ RDI-I } & $\mathrm{K}$ & 1343 & 36 & 72 \\
\hline & & $2 \mathrm{~K}$ & 860 & 27 & 54 \\
\hline & & Mean & 1102 & 32 & 64 \\
\hline & \multirow{3}{*}{ RDI-II } & $\mathrm{K}$ & 1030 & 30 & 60 \\
\hline & & $2 \mathrm{~K}$ & 935 & 28 & 56 \\
\hline & & Mean & 983 & 29 & 58 \\
\hline & \multirow{2}{*}{ Mean } & $\mathrm{K}$ & 1141 & 32 & 64 \\
\hline & & $2 \mathrm{~K}$ & 969 & 29 & 58 \\
\hline
\end{tabular}

${ }^{1} \mathrm{~K}$ (Control): 20 buds left for the first $500 \mathrm{~g}$ pruning weight and 10 more buds left for every additional $500 \mathrm{~g}$.
(20 kg/ha N; $30 \mathrm{~kg} / \mathrm{ha} \mathrm{P}_{2} \mathrm{O}_{5} ; 50 \mathrm{~kg} / \mathrm{ha} \mathrm{K}_{2} \mathrm{O}$ ) and at the end of May $(30 \mathrm{~kg} / \mathrm{ha} \mathrm{N})$.

The midday leaf water potentials (LWP) were measured in four leaves for each treatment using a portable pressure chamber device (Model 600 Pressure chamber, PMS instrument) between 11:30 hrs and 14:00 hrs. During the ripening period of grapes (when amount of total soluble solids (TSS) have reached approximately 22-23\%) $500 \mathrm{~g}$ berry samples with pedicels were taken from each replicate. These samples were maintained at $-80^{\circ} \mathrm{C}$ until the following analyses were performed.

For determination of sugars and organic acids, grape samples were prepared according to the method of Sturm et al. [22] and then analysed for the content of individual sugars (glucose, fructose and sucrose) and of organic acids (tartaric and malic acids). An Agilent 1100 HPLC system (Agilent Technologies, Palo Alto CA-USA) equipped with a pump system, a refractive index detector (RID) for sugar analysis, and a diode array detector (DAD) is monitored at $210 \mathrm{~nm}$ for the analysis of organic acids. Sugars and organic acids were simultaneously analyzed onto an Aminex HPX$87 \mathrm{H}$ column $(300 \times 7.8 \mathrm{~mm})($ Bio-Rad-UK) and kept at $55^{\circ} \mathrm{C}$. The analytical conditions used were as follows: flow $0.3 \mathrm{ml} / \mathrm{min}$, eluent $0.045 \mathrm{~N} \mathrm{H}_{2} \mathrm{SO}_{4}$ with $6 \%$ acetonitrile $(\mathrm{v} / \mathrm{v})$. The chromatographic peak corresponding to each sugar, and organic acid were identified by comparing the retention time with that of a standard. A calibration curve was prepared using the standards to determine the relationship between the peak area and concentration.

Extracts for analysis of phenolic compounds were prepared according to the method of Breksa et al. [23]. An Agilent 1100 HPLC system (Agilent Technologies, Palo Alto, CA, USA) with a diode array detector operated by Windows NT based ChemStation software was used for the phenolic compounds analysis. Separation was performed on a Beckman Ultrasphere ODS column (Roissy, France; $4.6 \mathrm{~mm} \times 250 \mathrm{~mm}, 5 \mu \mathrm{m})$. The mobile phase consisted of water with $5 \%$ formic acid (solvent $\mathrm{A} ; \mathrm{v} / \mathrm{v}$ ) and acetonitrile with $40 \%$ solvent A (solvent B; v/v). The elution program was performed as previously described [24]. The identification and assignation of each compounds was performed by comparing their retention times and UV spectra to authentic standards and also confirmed by an Agilent 6430LC-MS/ MS spectrometer equipped with an electrospray ionization source. The electrospray ionization mass spectrometry detection was performed in negative ion mode with the following optimized parameters: capillary temperature $400^{\circ} \mathrm{C}$, capillary voltage $-3 \mathrm{~V}$, nebulizer gas flow $1.75 \mathrm{~L} / \mathrm{min}$, desolvation gas flow $1 \mathrm{~L} / \mathrm{min}$, and spray voltage $5 \mathrm{kV}$. Analysis was performed in negative and positive mode [24].

Antioxidant activity analysis were performed according to the DPPH (2,2-diphenyl-1-picryl hydrazyl) method described by Kelebek et al. [24]. The absorbance was monitored at $517 \mathrm{~nm}$ by a UV-Visible spectrophotometer (Shimadzu UV-1201, Kyoto-Japan). Trolox calibration curve was used to calculate antioxidant activity of grape extracts and to express the antioxidant capacity in $\mathrm{mM}$ Trolox equivalent per $\mathrm{kg}$ of berry.

\subsection{Statistical analysis}

Variance analysis was performed according to the split plots experimental design with two replicates using SAS 
Table 2. Irrigation dates and water amount applied (L/vine) in Regulated Deficit Irrigation (RDI) treatments of Razak1 grapevine.

\begin{tabular}{|c|c|c|c|c|c|}
\hline \multirow{2}{*}{$\begin{array}{c}\text { Phenological } \\
\text { stages }\end{array}$} & \multirow{2}{*}{$\begin{array}{l}\text { Irrigation } \\
\text { dates }\end{array}$} & \multicolumn{2}{|c|}{2013 Year } & \multicolumn{2}{|c|}{2014 Year } \\
\hline & & RDI-I & RDI-II & RDI-I & RDI-II \\
\hline Bud break & & \multicolumn{2}{|c|}{24 April } & \multicolumn{2}{|c|}{23 April } \\
\hline Bloom & & \multicolumn{2}{|l|}{5 June } & \multicolumn{2}{|c|}{17 June } \\
\hline \multirow[t]{4}{*}{ Berry set } & & \multicolumn{2}{|c|}{16 June } & \multicolumn{2}{|c|}{24 June } \\
\hline & 17 July & 60.3 & 90.5 & \\
\hline & 26 July & 72 & 108 & & \\
\hline & 16 August & & & 28.4 & 42.5 \\
\hline Veraison & & \multicolumn{2}{|c|}{11 August } & \multicolumn{2}{|c|}{17 August } \\
\hline \multirow{3}{*}{$\begin{array}{l}\text { Total (Berry } \\
\text { set-Veraison) }\end{array}$} & & 132.3 & 198.5 & 28.4 & 42.5 \\
\hline & 19 August & 336.8 & 224.6 & & \\
\hline & 22 August & & & 72.2 & 48.2 \\
\hline Maturity & & \multicolumn{2}{|c|}{27 August } & \multicolumn{2}{|c|}{28 August } \\
\hline \multirow{5}{*}{$\begin{array}{l}\text { Total } \\
\text { (Veraison - } \\
\text { Maturity) } \\
\end{array}$} & & 336.8 & 224.6 & 72.2 & 48.2 \\
\hline & 29 August & 160.2 & 160.2 & 118.8 & 118.8 \\
\hline & 9 Sep. & & & 72.0 & 72.0 \\
\hline & $12 \mathrm{Sep}$. & 218.7 & 218.7 & & \\
\hline & 13 Sep. & & & 14.4 & 14.4 \\
\hline $\begin{array}{l}\text { Total (After } \\
\text { harvest) }\end{array}$ & & 378.9 & 378.9 & 205.2 & 205.2 \\
\hline \multicolumn{2}{|c|}{ GENERAL TOTAL } & 848 & 802 & 305.8 & 295.9 \\
\hline
\end{tabular}

statistical programmer, and least significant difference test (LSD) was used for separation of means of different treatments at $5 \%$ significance level.

\section{Results and discussion}

The water amounts applied in different periods according to applications are given in Table 2. As LWP values measured before flowering were $<-1.0 \mathrm{MPa}$, irrigation was not applied until berry set. Therefore we considered that the rainfall between September and December plus until berry set in 2013 and 2014 experimental years were sufficient $[1,20]$. A total rainfall of $679.6 \mathrm{~mm}$ and $500.8 \mathrm{~mm}$ were recorded in the experimental area between September and August in 2013 and 2014 (Table 3), respectively.

The effects of irrigation on the content of sugars and organic acids were significantly different (Table 4). However these differences varied by the years. Glucose, fructose and total sugar values for the first year (2013) in non-irrigated and for the second year (2014) in RDI-II experiments were higher than the other treatments. On the other hand, while malic and total acid amount was highest in RDI-II subject for 2013, tartaric and total acid values were the highest in non-irrigated ones. Glucose and fructose values were close to each other in all applications. The glucose and fructose ratio was slightly higher than 1 as mentioned in previous studies [14,20]. Contrary to the researchers stating that berry growth in grapevines to which excess bud load was applied was lower compared to grapevines with less load as a result of the available sugar amount per each berry and water absorption decrease of berries $[1,20]$, in this study, no significant difference was observed between bud load applications except for sucrose. It was determined that sucrose amount was very low compared with other sugar components. The values given in Table 4 have shown that a certain amount of increase in acidity occurred due to high bud load application. Tartaric

Table 3. Total rainfall between September, 2012 and August, 2014 in experimental area (Adana Sixth Regional Directorate of Meteorology, Pozantı Climate station).

\begin{tabular}{|c|c|c|c|c|c|c|c|}
\hline Years & Months & $\begin{array}{c}\text { Total rainfall } \\
\quad(\mathbf{m m})\end{array}$ & Total (mm) & Years & Months & $\begin{array}{c}\text { Total rainfall } \\
\quad(\mathbf{m m})\end{array}$ & Total (mm) \\
\hline \multirow{4}{*}{2012} & September & 0.4 & \multirow{4}{*}{432.6} & \multirow{4}{*}{2013} & September & 1.8 & \multirow{4}{*}{62} \\
\hline & October & 107.0 & & & October & 30.4 & \\
\hline & November & 93.4 & & & November & 10.4 & \\
\hline & December & 231.8 & & & December & 19.4 & \\
\hline \multirow{8}{*}{2013} & January & 31.4 & \multirow{5}{*}{234.8} & \multirow{8}{*}{2014} & January & 94.8 & \multirow{5}{*}{360} \\
\hline & February & 47.2 & & & February & 10.6 & \\
\hline & March & 65.6 & & & March & 124.8 & \\
\hline & April & 67.6 & & & April & 42.6 & \\
\hline & May & 23.0 & & & May & 87.2 & \\
\hline & June & 11.8 & \multirow{2}{*}{12.2} & & June & 76.8 & \multirow{3}{*}{78.8} \\
\hline & July & 0.4 & & & July & 0.6 & \\
\hline & August & 0 & & & August & 1.4 & \\
\hline \multicolumn{2}{|l|}{ Total } & 679.6 & 679.6 & \multicolumn{2}{|l|}{ Total } & 500.8 & 500.8 \\
\hline
\end{tabular}


Table 4. Effects of two Regulated Deficit Irrigation (RDI) practices and two bud load levels on sugar and organic acid contents of Razakı $(\mathrm{g} / \mathrm{kg})$.

\begin{tabular}{|c|c|c|c|c|c|c|c|c|c|c|c|c|c|c|}
\hline \multirow[t]{2}{*}{$\begin{array}{l}\text { Source of } \\
\text { variance }^{1}\end{array}$} & \multicolumn{2}{|c|}{ Glucose $^{1}$} & \multicolumn{2}{|c|}{ Fructose } & \multicolumn{2}{|c|}{ Sucrose } & \multicolumn{2}{|c|}{ Total sugars } & \multicolumn{2}{|c|}{ Tartaric acid } & \multicolumn{2}{|c|}{ Malic acid } & \multicolumn{2}{|c|}{$\begin{array}{c}\text { Total organic } \\
\text { acids }\end{array}$} \\
\hline & 2013 & 2014 & 2013 & 2014 & 2013 & 2014 & 2013 & 2014 & 2013 & 2014 & 2013 & 2014 & 2013 & 2014 \\
\hline \multicolumn{15}{|l|}{ Irrigation } \\
\hline $\begin{array}{l}\text { No } \\
\text { irrigation }\end{array}$ & $100.37 \mathrm{a}$ & $84.89 \mathrm{c}$ & $97.69 \mathrm{a}$ & $75.82 \mathrm{c}$ & $0.80 \mathrm{~b}$ & $2.59 a$ & $198.86 a$ & 163.30c & 4.48 & $5.47 \mathrm{a}$ & $2.13 \mathrm{c}$ & $2.34 \mathrm{c}$ & $6.61 b$ & $7.81 \mathrm{a}$ \\
\hline RDI-I & $91.66 \mathrm{~b}$ & $87.69 \mathrm{~b}$ & $90.10 \mathrm{~b}$ & $78.65 b$ & $1.09 \mathrm{a}$ & $2.48 \mathrm{a}$ & $182.85 \mathrm{~b}$ & $168.82 b$ & 4.38 & $4.27 \mathrm{~b}$ & $2.29 \mathrm{~b}$ & $2.51 \mathrm{~b}$ & $6.67 \mathrm{~b}$ & $6.78 \mathrm{~b}$ \\
\hline RDI-II & $90.97 \mathrm{~b}$ & $92.49 \mathrm{a}$ & $89.38 \mathrm{~b}$ & $85.45 a$ & $1.24 \mathrm{a}$ & $2.00 \mathrm{~b}$ & $181.59 \mathrm{~b}$ & $179.94 a$ & 4.43 & $3.63 \mathrm{c}$ & $2.67 \mathrm{a}$ & $2.59 \mathrm{a}$ & $7.10 \mathrm{a}$ & $6.22 \mathrm{c}$ \\
\hline LSD 5\% & 1.60 & 1.02 & 1.68 & 1.31 & 0.10 & 0.24 & 2.94 & 1.47 & NS & 0.33 & 0.06 & 0.10 & 0.22 & 0.28 \\
\hline \multicolumn{15}{|c|}{ Bud load level } \\
\hline $\mathrm{K}$ & 94.94 & 88.30 & 92.97 & 80.29 & $0.54 \mathrm{~b}$ & $2.52 \mathrm{a}$ & 188.45 & 171.11 & $4.18 \mathrm{~b}$ & 4.35 & $2.41 \mathrm{a}$ & $2.54 \mathrm{a}$ & $6.59 \mathrm{~b}$ & 6.89 \\
\hline $2 \mathrm{~K}$ & 93.73 & 88.41 & 91.81 & 79.66 & $1.55 \mathrm{a}$ & $2.20 \mathrm{~b}$ & 187.10 & 170.26 & $4.68 \mathrm{a}$ & 4.57 & $2.31 \mathrm{~b}$ & $2.41 \mathrm{~b}$ & $6.99 a$ & 6.98 \\
\hline LSD 5\% & NS & NS & NS & NS & 0.08 & 0.20 & NS & NS & 0.07 & NS & 0.05 & 0.08 & 0.18 & NS \\
\hline \multicolumn{15}{|l|}{ Interaction } \\
\hline No irr. $\times K$ & $101.66 \mathrm{a}$ & $92.92 \mathrm{~b}$ & 99.09a & $84.37 \mathrm{~b}$ & $1.61 \mathrm{c}$ & $2.49 \mathrm{~b}$ & $202.36 a$ & $179.78 b$ & $5.02 \mathrm{ab}$ & $4.26 \mathrm{c}$ & $2.09 \mathrm{~d}$ & $2.66 \mathrm{ab}$ & $7.10 \mathrm{~b}$ & $6.92 \mathrm{c}$ \\
\hline No irr. $\times 2 \mathrm{~K}$ & $99.09 \mathrm{a}$ & $76.86 \mathrm{f}$ & 96.28ab & $67.28 \mathrm{e}$ & $0.00 \mathrm{~d}$ & $2.69 \mathrm{ab}$ & $195.37 b$ & $146.83 f$ & $3.95 \mathrm{~d}$ & $6.67 a$ & $2.17 \mathrm{~d}$ & $2.02 \mathrm{~d}$ & $6.12 c$ & $8.69 a$ \\
\hline RDI-I $\times \mathrm{K}$ & $94.87 \mathrm{~b}$ & $83.97 \mathrm{e}$ & $93.43 \mathrm{bc}$ & $75.45 \mathrm{~d}$ & $0.00 \mathrm{~d}$ & $2.97 \mathrm{a}$ & $188.30 \mathrm{c}$ & $162.40 \mathrm{e}$ & $3.25 \mathrm{e}$ & $5.13 b$ & $2.40 \mathrm{c}$ & $2.50 \mathrm{c}$ & $5.65 \mathrm{~d}$ & $7.63 b$ \\
\hline RDI-I $\times 2 \mathrm{~K}$ & $88.45 \mathrm{c}$ & $91.41 \mathrm{c}$ & $86.78 \mathrm{~d}$ & $81.86 \mathrm{c}$ & $2.19 \mathrm{~b}$ & $1.99 \mathrm{c}$ & \begin{tabular}{|l|}
$177.41 \mathrm{~d}$ \\
\end{tabular} & $175.25 \mathrm{c}$ & $5.50 \mathrm{a}$ & $3.40 \mathrm{~d}$ & $2.18 \mathrm{~d}$ & $2.53 \mathrm{bc}$ & 7.69a & $5.93 \mathrm{~d}$ \\
\hline RDI-II $\times \mathrm{K}$ & $88.28 \mathrm{c}$ & $88.01 \mathrm{~d}$ & $86.40 \mathrm{~d}$ & $81.06 \mathrm{c}$ & $0.00 \mathrm{~d}$ & $2.09 \mathrm{c}$ & $174.68 d$ & 171.16d & $4.27 \mathrm{~cd}$ & $3.65 \mathrm{~d}$ & $2.75 a$ & $2.47 \mathrm{c}$ & $7.02 \mathrm{~b}$ & $6.12 d$ \\
\hline RDI-II $\times 2 \mathrm{~K}$ & $93.66 \mathrm{~b}$ & $96.97 \mathrm{a}$ & $92.37 \mathrm{c}$ & $89.84 a$ & $2.47 \mathrm{a}$ & $1.91 \mathrm{c}$ & $188.51 \mathrm{c}$ & $188.72 \mathrm{a}$ & $4.59 \mathrm{bc}$ & $3.62 \mathrm{~d}$ & $2.59 \mathrm{~b}$ & $2.70 \mathrm{a}$ & $7.18 \mathrm{~b}$ & $6.32 \mathrm{~d}$ \\
\hline LSD 5\% & 2.27 & 1.45 & 2.37 & 1.85 & 0.15 & 0.34 & 4.16 & 2.07 & 0.38 & 0.47 & 0.08 & 0.15 & 0.31 & 0.40 \\
\hline
\end{tabular}

${ }^{1}$ Means followed by different letters on the same column are significantly different according to LSD at $P<0.05$. NS. Not significant.

acid values were higher than malic acid in all applications. Such conclusions were also reached by many researchers before $[1,14,20]$. It was observed that irrigation applications did not change this situation. Irrigation and bud load interactions were statistically significant in all sugars and organic acid properties examined.

Thirteen different phenolic compounds (PC), including two flavanols, six phenolic acids, and five flavonols were identified in Razakı berries (Tables 5-7). Considering these compounds differences between application effects and interactions were statistically significant. Besides catechin and epicatechin, rutin, myricetin-3-glucoside total $\mathrm{PC}$ values were highest in rainfed application.

Total and PC values, except gallic and caffeic acid was higher in $2 \mathrm{~K}$ application. The total PC values ranged 2013 and 2014 respectively from 161.72 and $173.63 \mathrm{mg} / \mathrm{kg}$ (in RDI-I 2K) to 542.96 and $570.63 \mathrm{mg} / \mathrm{kg}$ (in No irrigation $2 \mathrm{~K})$. The total value of berry skin phenolics of Syrah was stated as between 1878 and $2478 \mu \mathrm{g} / \mathrm{g}$ by Wessner and Kurtural [25] and detected by Kurtural et al. [26] as between 2170 and $2548 \mu \mathrm{g} / \mathrm{g}$. Kelebek [14] detected non-coloured phenolic compounds of the wines obtained from Kalecik karasi, Okuzgozu and Bogazkere grapes. In his study, the phenolic amounts of the studied varieties changed between $142 \mathrm{mg} / \mathrm{L}$ and $349 \mathrm{mg} / \mathrm{L}$. According to the Göktürk Baydar et al. [27], total phenolic contents varied from 217 to $1336 \mathrm{mg} / \mathrm{L}$ in wines from Cabernet Sauvignon, Kalecik Karası and Narince grape cultivars. Catechin and epicatechin were the most important elements among phenolic compounds [10,28,29].

Antioxidant activity level was higher in RDI-I and $2 \mathrm{~K}$ treatments (Table 8 ) in 2013. However there were no significant differences among the treatments in 2014.
Table 5. Effects of two Regulated Deficit Irrigation (RDI) practices and two bud load levels on flavanol contents $(\mathrm{mg} / \mathrm{kg})$ of Razakı.

\begin{tabular}{|l|l|l|l|l|}
\hline $\begin{array}{c}\text { Source of } \\
\text { variance }\end{array}$ & \multicolumn{5}{|c|}{ Catechin $^{1}$} & \multicolumn{2}{c|}{ Epicatechin } \\
\hline & 2013 & 2014 & 2013 & 2014 \\
\hline Irrigation & \multicolumn{5}{|l|}{} \\
\hline No irrigation & $141.64 \mathrm{a}$ & $153.76 \mathrm{a}$ & $197.00 \mathrm{a}$ & $197.86 \mathrm{a}$ \\
\hline RDI-I & $77.73 \mathrm{~b}$ & $84.39 \mathrm{~b}$ & $114.58 \mathrm{c}$ & $115.08 \mathrm{c}$ \\
\hline RDI-II & $58.24 \mathrm{c}$ & $61.14 \mathrm{c}$ & $119.32 \mathrm{~b}$ & $118.95 \mathrm{~b}$ \\
\hline LSD 5\% & 0.81 & 2.68 & 0.87 & 2.66 \\
\hline Bud load level & $72.86 \mathrm{~b}$ & $77.71 \mathrm{~b}$ & $126.54 \mathrm{~b}$ & $126.50 \mathrm{~b}$ \\
\hline K & $112.22 \mathrm{a}$ & $121.82 \mathrm{a}$ & $160.73 \mathrm{a}$ & $161.42 \mathrm{a}$ \\
\hline $2 \mathrm{~K}$ & 0.66 & 2.19 & 0.71 & 2.17 \\
\hline LSD 5\% & \multicolumn{5}{|l}{} \\
\hline Interaction & & & & \\
\hline No irr. $\times \mathrm{K}$ & $56.71 \mathrm{~d}$ & $61.56 \mathrm{c}$ & $111.10 \mathrm{e}$ & $111.58 \mathrm{~d}$ \\
\hline No irr. $\times 2 \mathrm{~K}$ & $226.57 \mathrm{a}$ & $245.95 \mathrm{a}$ & $282.91 \mathrm{a}$ & $284.13 \mathrm{a}$ \\
\hline RDI-I $\times \mathrm{K}$ & $100.43 \mathrm{~b}$ & $109.02 \mathrm{~b}$ & $153.52 \mathrm{~b}$ & $154.18 \mathrm{~b}$ \\
\hline RDI-I $\times 2 \mathrm{~K}$ & $55.04 \mathrm{e}$ & $59.75 \mathrm{c}$ & $75.65 \mathrm{f}$ & $75.97 \mathrm{e}$ \\
\hline RDI-II $\times \mathrm{K}$ & $61.44 \mathrm{c}$ & $62.53 \mathrm{c}$ & $115.00 \mathrm{~d}$ & $113.74 \mathrm{~d}$ \\
\hline RDI-II $\times 2 \mathrm{~K}$ & $55.04 \mathrm{e}$ & $59.75 \mathrm{c}$ & $123.63 \mathrm{c}$ & $124.17 \mathrm{c}$ \\
\hline LSD $5 \%$ & 1.15 & 3.79 & 1.23 & 3.76 \\
\hline
\end{tabular}

${ }^{1}$ Means followed by different letters on the same column are significantly different according to LSD at $P<0.05$. 
$38^{\text {th }}$ World Congress of Vine and Wine

Table 6. Effects of two Regulated Deficit Irrigation (RDI) practices and two bud load levels on phenol acids (mg/kg) contents of Razak1.

\begin{tabular}{|c|c|c|c|c|c|c|c|c|c|c|c|c|}
\hline \multirow[t]{2}{*}{$\begin{array}{l}\text { Source of } \\
\text { variance }^{1}\end{array}$} & \multicolumn{2}{|c|}{ Gallic acid } & \multicolumn{2}{|c|}{$\begin{array}{c}\text { Protocatechuic } \\
\text { acid }\end{array}$} & \multicolumn{2}{|c|}{$\begin{array}{c}\text { Trans caftaric } \\
\text { acid }\end{array}$} & \multicolumn{2}{|c|}{$\begin{array}{c}\text { Trans coutaric } \\
\text { acid }\end{array}$} & \multicolumn{2}{|c|}{ Caffeic acid } & \multicolumn{2}{|c|}{$p$-Coumaric acid } \\
\hline & 2013 & 2014 & 2013 & 2014 & 2013 & 2014 & 2013 & 2014 & 2013 & 2014 & 2013 & 2014 \\
\hline \multicolumn{13}{|l|}{ Irrigation } \\
\hline No irrigation & $5.20 \mathrm{c}$ & $4.49 \mathrm{c}$ & $3.12 b$ & $2.37 \mathrm{~b}$ & $12.45 b$ & $19.58 \mathrm{~b}$ & $4.98 \mathrm{~b}$ & $5.91 \mathrm{~b}$ & $0.49 \mathrm{c}$ & $0.52 \mathrm{c}$ & $0.61 \mathrm{a}$ & $0.65 a$ \\
\hline RDI-I & $7.56 \mathrm{a}$ & $6.52 \mathrm{a}$ & $2.12 \mathrm{c}$ & $1.61 \mathrm{c}$ & $9.54 \mathrm{c}$ & $15.01 \mathrm{c}$ & $4.89 \mathrm{c}$ & $5.81 \mathrm{~b}$ & $0.78 \mathrm{a}$ & $0.84 \mathrm{a}$ & $0.00 \mathrm{~b}$ & $0.00 \mathrm{~b}$ \\
\hline RDI-II & $5.33 b$ & $4.60 \mathrm{~b}$ & $4.36 \mathrm{a}$ & $3.32 \mathrm{a}$ & $18.14 \mathrm{a}$ & $28.54 \mathrm{a}$ & $6.98 \mathrm{a}$ & $8.28 \mathrm{a}$ & $0.75 b$ & $0.80 \mathrm{~b}$ & $0.00 \mathrm{~b}$ & $0.00 \mathrm{~b}$ \\
\hline LSD 5\% & 0.006 & 0.01 & 0.02 & 0.05 & 0.08 & 0.36 & 0.03 & 0.10 & 0.005 & 0.02 & 0.0006 & 0.002 \\
\hline \multicolumn{13}{|l|}{ Bud load level } \\
\hline $\mathrm{K}$ & $6.05 \mathrm{a}$ & $5.22 \mathrm{a}$ & $2.94 b$ & $2.24 b$ & $12.20 \mathrm{~b}$ & $19.19 \mathrm{~b}$ & $4.13 b$ & $4.90 \mathrm{~b}$ & $0.78 \mathrm{a}$ & $0.83 a$ & $0.22 \mathrm{a}$ & $0.24 \mathrm{a}$ \\
\hline $2 \mathrm{~K}$ & $6.01 \mathrm{~b}$ & $5.18 \mathrm{~b}$ & $3.45 \mathrm{a}$ & $2.63 \mathrm{a}$ & $14.56 \mathrm{a}$ & $22.90 \mathrm{a}$ & $7.10 \mathrm{a}$ & $8.42 \mathrm{a}$ & $0.57 \mathrm{~b}$ & $0.60 \mathrm{~b}$ & $0.18 \mathrm{~b}$ & $0.19 \mathrm{~b}$ \\
\hline LSD 5\% & 0.005 & 0.01 & 0.02 & 0.04 & 0.06 & 0.29 & 0.02 & 0.08 & 0.004 & 0.01 & 0.0005 & 0.002 \\
\hline \multicolumn{13}{|l|}{ Interaction } \\
\hline No irr. $\times K$ & $5.58 \mathrm{c}$ & $4.81 \mathrm{c}$ & $4.36 \mathrm{~b}$ & $3.32 \mathrm{~b}$ & $16.70 \mathrm{~b}$ & $26.27 \mathrm{~b}$ & $3.78 \mathrm{f}$ & $4.49 \mathrm{f}$ & $0.97 \mathrm{a}$ & $1.04 \mathrm{a}$ & $0.67 \mathrm{a}$ & $0.72 \mathrm{a}$ \\
\hline No irr. $\times 2 \mathrm{~K}$ & $4.83 \mathrm{f}$ & $4.16 \mathrm{f}$ & $1.87 \mathrm{e}$ & $1.42 \mathrm{e}$ & $8.20 \mathrm{f}$ & $12.90 \mathrm{f}$ & $6.17 \mathrm{~b}$ & $7.32 \mathrm{~b}$ & $0.00 \mathrm{f}$ & $0.00 \mathrm{f}$ & $0.54 \mathrm{~b}$ & $0.57 \mathrm{~b}$ \\
\hline RDI-I $\times \mathrm{K}$ & $7.10 \mathrm{~b}$ & $6.12 b$ & $1.86 \mathrm{e}$ & $1.42 \mathrm{e}$ & $8.65 \mathrm{e}$ & $13.60 \mathrm{e}$ & $4.43 \mathrm{~d}$ & $5.26 \mathrm{~d}$ & $0.74 \mathrm{~d}$ & $0.78 \mathrm{~d}$ & $0.00 \mathrm{c}$ & $0.00 \mathrm{c}$ \\
\hline RDI-I $\times 2 \mathrm{~K}$ & $8.01 \mathrm{a}$ & $6.91 \mathrm{a}$ & $2.38 \mathrm{~d}$ & $1.81 \mathrm{~d}$ & $10.43 \mathrm{~d}$ & $16.41 \mathrm{~d}$ & $5.35 \mathrm{c}$ & $6.36 \mathrm{c}$ & $0.83 \mathrm{c}$ & $0.89 \mathrm{c}$ & $0.00 \mathrm{c}$ & $0.00 \mathrm{c}$ \\
\hline RDI-II $\times \mathrm{K}$ & $5.47 \mathrm{~d}$ & $4.71 \mathrm{~d}$ & $2.61 \mathrm{c}$ & $1.98 \mathrm{c}$ & $11.25 \mathrm{c}$ & $17.69 \mathrm{c}$ & $4.18 \mathrm{e}$ & $4.97 \mathrm{e}$ & $0.64 \mathrm{e}$ & $0.68 \mathrm{e}$ & $0.00 \mathrm{c}$ & $0.00 \mathrm{c}$ \\
\hline $\mathrm{RDI}-\mathrm{II} \times 2 \mathrm{~K}$ & $5.20 \mathrm{e}$ & $4.48 \mathrm{e}$ & $6.12 \mathrm{a}$ & $4.65 \mathrm{a}$ & $25.04 \mathrm{a}$ & $39.39 a$ & $9.77 \mathrm{a}$ & $11.60 \mathrm{a}$ & $0.87 \mathrm{~b}$ & $0.93 b$ & $0.00 \mathrm{c}$ & $0.00 \mathrm{c}$ \\
\hline LSD 5\% & 0.008 & 0.02 & 0.03 & 0.07 & 0.11 & 0.51 & 0.04 & 0.14 & 0.007 & 0.02 & 0.0009 & 0.003 \\
\hline
\end{tabular}

${ }^{1}$ Means followed by different letters on the same column are significantly different according to LSD at $P<0.05$.

Table 7. Effects of two Regulated Deficit Irrigation (RDI) practices and two bud load levels on content of flavonols and total phenolic compounds $(\mathrm{mg} / \mathrm{kg})$ of Razak1.

\begin{tabular}{|c|c|c|c|c|c|c|c|c|c|c|c|c|}
\hline \multirow[t]{2}{*}{$\begin{array}{l}\text { Source of } \\
\text { variance }^{1}\end{array}$} & \multicolumn{2}{|c|}{ Ferulik asit } & \multicolumn{2}{|c|}{ Rutin $^{1}$} & \multicolumn{2}{|c|}{$\begin{array}{l}\text { Myricetin } \\
\text { 3-glucoside }\end{array}$} & \multicolumn{2}{|c|}{$\begin{array}{l}\text { Isoramnetin } \\
\text { 3-glucoside }\end{array}$} & \multicolumn{2}{|c|}{$\begin{array}{l}\text { Quercetin } \\
\text { 3-glucoside }\end{array}$} & \multicolumn{2}{|c|}{ Total phenols } \\
\hline & 2013 & 2014 & 2013 & 2014 & 2013 & 2014 & 2013 & 2014 & 2013 & 2014 & 2013 & 2014 \\
\hline \multicolumn{13}{|l|}{ Irrigation } \\
\hline No irrigation & $0.32 \mathrm{c}$ & $0.34 \mathrm{c}$ & $1.11 \mathrm{a}$ & $1.18 \mathrm{a}$ & $5.54 \mathrm{a}$ & $5.90 \mathrm{a}$ & $0.47 \mathrm{~b}$ & $0.52 \mathrm{~b}$ & 0.00 & $1.29 \mathrm{~b}$ & $372.90 \mathrm{a}$ & $394.34 \mathrm{a}$ \\
\hline RDI-I & $0.47 \mathrm{a}$ & $0.50 \mathrm{a}$ & $0.53 b$ & $0.57 \mathrm{~b}$ & $2.59 \mathrm{c}$ & $2.76 \mathrm{c}$ & $0.50 \mathrm{a}$ & $0.55 \mathrm{a}$ & 0.00 & $1.38 \mathrm{a}$ & $221.31 b$ & $235.01 \mathrm{~b}$ \\
\hline RDI-II & $0.46 \mathrm{~b}$ & $0.49 \mathrm{~b}$ & $0.39 \mathrm{c}$ & $0.41 \mathrm{c}$ & $3.06 \mathrm{~b}$ & $3.26 \mathrm{~b}$ & $0.40 \mathrm{c}$ & $0.45 \mathrm{c}$ & 0.00 & $1.11 \mathrm{c}$ & $217.44 \mathrm{c}$ & $234.32 \mathrm{~b}$ \\
\hline LSD 5\% & 0.002 & 0.006 & 0.006 & 0.02 & 0.03 & 0.12 & 0.001 & 0.003 & & 0.008 & 1.67 & 5.26 \\
\hline \multicolumn{13}{|l|}{ Bud load level } \\
\hline $\mathrm{K}$ & $0.38 \mathrm{~b}$ & $0.41 \mathrm{~b}$ & $0.53 \mathrm{~b}$ & $0.56 \mathrm{~b}$ & $2.27 \mathrm{~b}$ & $2.41 \mathrm{~b}$ & $0.43 \mathrm{~b}$ & $0.48 \mathrm{~b}$ & 0.00 & $1.19 \mathrm{~b}$ & $229.34 b$ & $243.86 \mathrm{~b}$ \\
\hline $2 \mathrm{~K}$ & $0.45 \mathrm{a}$ & $0.48 \mathrm{a}$ & $0.82 \mathrm{a}$ & $0.88 \mathrm{a}$ & $5.20 \mathrm{a}$ & $5.54 \mathrm{a}$ & $0.48 \mathrm{a}$ & $0.53 \mathrm{a}$ & 0.00 & $1.33 \mathrm{a}$ & $311.76 \mathrm{a}$ & $331.93 \mathrm{a}$ \\
\hline LSD 5\% & 0.002 & 0.005 & 0.005 & 0.02 & 0.03 & 0.09 & 0.0008 & 0.002 & & 0.006 & 1.36 & 4.29 \\
\hline \multicolumn{13}{|l|}{ Interaction } \\
\hline No irr. $\times K$ & $0.15 \mathrm{f}$ & $0.16 \mathrm{f}$ & $0.49 \mathrm{e}$ & $0.52 \mathrm{c}$ & $1.93 \mathrm{f}$ & $2.05 \mathrm{e}$ & $0.39 \mathrm{e}$ & $0.43 \mathrm{e}$ & 0.00 & $1.08 \mathrm{e}$ & $202.85 d$ & $218.05 \mathrm{~d}$ \\
\hline No irr. $\times 2 \mathrm{~K}$ & $0.48 \mathrm{c}$ & $0.51 \mathrm{c}$ & $1.73 \mathrm{a}$ & $1.84 \mathrm{a}$ & $9.14 \mathrm{a}$ & $9.74 \mathrm{a}$ & $0.54 \mathrm{~b}$ & $0.60 \mathrm{~b}$ & 0.00 & $1.49 \mathrm{~b}$ & $542.96 \mathrm{a}$ & $570.63 \mathrm{a}$ \\
\hline RDI-I $\times \mathrm{K}$ & $0.58 \mathrm{a}$ & $0.62 \mathrm{a}$ & $0.56 \mathrm{~b}$ & $0.59 \mathrm{~b}$ & $2.48 \mathrm{~d}$ & $2.64 d$ & $0.55 \mathrm{a}$ & $0.61 \mathrm{a}$ & 0.00 & $1.53 \mathrm{a}$ & $280.90 \mathrm{~b}$ & $296.39 \mathrm{~b}$ \\
\hline RDI-I $\times 2 \mathrm{~K}$ & $0.36 \mathrm{e}$ & $0.39 \mathrm{e}$ & $0.51 \mathrm{~d}$ & $0.54 \mathrm{c}$ & $2.70 \mathrm{c}$ & $2.88 \mathrm{c}$ & $0.45 \mathrm{~d}$ & $0.49 \mathrm{~d}$ & 0.00 & $1.23 \mathrm{~d}$ & $161.72 \mathrm{e}$ & $173.63 \mathrm{e}$ \\
\hline RDI-II $\times \mathrm{K}$ & $0.41 \mathrm{~d}$ & $0.43 \mathrm{~d}$ & $0.54 \mathrm{c}$ & $0.57 \mathrm{bc}$ & $2.39 \mathrm{e}$ & $2.54 \mathrm{~d}$ & $0.35 \mathrm{f}$ & $0.39 \mathrm{f}$ & 0.00 & $0.97 \mathrm{f}$ & $204.27 d$ & $217.14 d$ \\
\hline RDI-II $\times 2 \mathrm{~K}$ & $0.51 \mathrm{~b}$ & $0.54 \mathrm{~b}$ & $0.24 \mathrm{f}$ & $0.25 \mathrm{~d}$ & $3.74 \mathrm{~b}$ & $3.99 \mathrm{~b}$ & $0.46 \mathrm{c}$ & $0.50 \mathrm{c}$ & 0.00 & $1.26 \mathrm{c}$ & $230.61 \mathrm{c}$ & $251.51 \mathrm{c}$ \\
\hline LSD 5\% & 0.003 & 0.008 & 0.008 & 0.03 & 0.05 & 0.16 & 0.001 & 0.004 & & 0.01 & 2.36 & 7.43 \\
\hline
\end{tabular}

${ }^{1}$ Means followed by different letters on the same column are significantly different according to LSD at $P<0.05$. 
Table 8. Effects of two Regulated Deficit Irrigation (RDI) practices and two bud load levels on antioxidant activity (mM Trolox/ $\mathrm{kg})$ of Razak1.

\begin{tabular}{|l|l|l|}
\hline \multicolumn{2}{|l|}{ Source of variance ${ }^{1}$} & \multicolumn{2}{|c|}{ Antioxidant activity } \\
\hline \multicolumn{2}{|l|}{2013} & 2014 \\
\hline Irrigation & $305.18 \mathrm{~b}$ & 381.71 \\
\hline No irrigation & $309.49 \mathrm{a}$ & 433.21 \\
\hline RDI-I & $305.38 \mathrm{~b}$ & 468.38 \\
\hline RDI-II & 1.11 & NS \\
\hline LSD 5\% & \multicolumn{3}{|l|}{} \\
\hline Bud load level & $305.67 \mathrm{~b}$ & 406.75 \\
\hline K & $307.69 \mathrm{a}$ & 448.78 \\
\hline $2 \mathrm{~K}$ & 0.90 & NS \\
\hline LSD 5\% & & \\
\hline Interaction & $308.45 \mathrm{~b}$ & 368.83 \\
\hline No irr. $\times \mathrm{K}$ & $301.90 \mathrm{e}$ & 394.58 \\
\hline No irr. $\times 2 \mathrm{~K}$ & $302.90 \mathrm{de}$ & 409.83 \\
\hline RDI-I $\times \mathrm{K}$ & $316.08 \mathrm{a}$ & 456.58 \\
\hline RDI-I $\times 2 \mathrm{~K}$ & $305.65 \mathrm{c}$ & 441.58 \\
\hline RDI-II $\times \mathrm{K}$ & $305.10 \mathrm{~cd}$ & 495.17 \\
\hline RDI-II $\times 2 \mathrm{~K}$ & 1.57 & NS \\
\hline LSD $5 \%$ & \multicolumn{2}{|l}{} \\
\hline
\end{tabular}

${ }^{1}$ Means followed by different letters on the same column are significantly different according to LSD at $P<0.05$. NS. Not significant.

Results indicated that applying deficit irrigation did not change the antioxidant activity in grapes.

The effect of RDI changes according to phenological stage and the level of applied irrigation stress [5], and the berry and wine quality may be enhanced [10]. Bowen et al. [4] and Keller et al. [3] stated in their studies that fruit composition, anthocyanin and berry content had been effected slightly or inconsistently from deficit irrigation and yield load balance and that the effect varied according to years or seasonal changes. Benismail et al. [30] on Cardinal; Fawzi et al. [31] on Seedless grape variety determined that the vegetative growth decreased depending on bud load increase per grapevine, while there were no change in the biochemical contents in grapes.

\section{Conclusion}

The overall findings of the present study indicate that RDI-I strategy, applying lower amount of water between berry set and veraison, can be used for maintaining better quality and yield of grapes. The application of $2 \mathrm{~K}$ bud load together with the deficit irrigation practice (RDI-I) did not change significantly investigated quality attributes of the berries. Findings of this study showed that in addition to an increase in grape yield due to higher bud load practice, optimum values from essential compounds could also be obtained with a supplementary irrigation application using regulated deficit irrigation.
The authors thank TUBITAK (Scientific and Technological Research Council of Turkey) for the financial support provided for this study (Project number: 112O059).

\section{References}

[1] S. Çelik, Namık Kemal Üniv. Ziraat Fak. Bahçe Bitkileri Böl. Tekirdağ. Avcı Ofset, Istanbul. 428 s. (2007)

[2] A.R. Basinger, E.W. Hellman, Int. J. of Fruit Sci. 6, 3-22 (2006)

[3] M. Keller, R.P. Smithyman, L.J. Mills, Am. J. Enol. Vitic. 59, 221-234 (2008)

[4] P. Bowen, C. Bogdanoff, K. Kevin Usher, B. Estergaard, M. Watson, Am. J. Enol. Vitic. 62, 9-22 (2011)

[5] P.E. Kriedemann, I. Goodwin, Irrigation Insights No. 4. Land and Water Australia, Canberra (2003)

[6] M.M. Chaves, T.P. Santos, C.R. Souza, M.F. Ortuño, M.L. Rodrigues, C.M. Lopes, J.P. Maroco, J.S. Pereira, Ann. Appl. Biol. 150, 237-252 (2007)

[7] D.B. Terry, S.K. Kurtural, Am. J. Enol. Vitic. 62, 426-437 (2011)

[8] K.C. Shellie, Am. J. Enol. Vitic. 57, 514-518 (2006)

[9] R. Ferreyra, G. Selles, J. Peralta, L. Burgos, J. Valenzuela, Agricultura Tecnica, 62, 406-417 (2002)

[10] J.A. Kennedy, M.A. Matthews, A.L. Waterhouse, Am. J. Enol. Vitic. 53, 268-274 (2002)

[11] P. Romero, J.I. Fernandez-Fernandez, A. MartinezCutillas, Am. J. Enol. Vitic. 61, 300-312 (2010)

[12] H. Ojeda, C. Andary, E. Kraeva, A. Carbonneau, A. Deloire, Am. J. Enol. Vitic. 53, 4, 261-267 (2002)

[13] M.L. De La Orts, A. Martinez-Cutillas, J.M. LopezRoca, E. Gomez-Plaza, Spanish J. of Agric. Res. 3, 352-361 (2005)

[14] H. Kelebek, Çukurova Üniv. Fen Bilimleri Enst. Doktora Tezi. Adana. 278 s (2009)

[15] S. Sério, M.D. Rivero-Pérez, A.C. Correia, A.M. Jordão, M.L. González-San José, Ciência Téc. Vitiv. 29, 1, 1-8 (2014)

[16] S. Gök Tangolar, E. Kafkas, S. Tangolar, VII. Türkiye Bağcılık ve Teknolojileri Sempozyumu, 5-9 Ekim Manisa-Salihli (2009)

[17] E.S. Pinheiro, J.M. Correia da Costa, E. Clemente, J. of Food Tech. 7, 3, 78-83 (2009)

[18] A. Sabır, E. Kafkas, S. Tangolar, Spanish J. of Agric. Res. 8, 2, 425-433 (2010)

[19] B. Basile, J. Marsal, M. Mata, X. Vallverdú, J. Bellvert, J. Girona, Am. J. Enol. Vitic. 62, 452-461 (2011)

[20] A.J. Winkler, J.A. Cook, W.M. Kliewer, L.A. Lider, Univ. of California Press. Berkeley, Los Angeles and London, p. 710 (1974)

[21] M. Ahmedullah, D.G. Himmelrick, (Editors; D. G. Himmelrick, G. J. Galletta). The Haworth press. 10 alice street Binghapton, NewYork, 383-471 (1990)

[22] K. Sturm, D. Koron, F. Stampar, Food Chem. 83, 417-422 (2003)

[23] A.P. Breksa, G.R. Takeoka, M.B. Hidalgo, A. Vilches, J. Vasse, D.W. Ramming, Food Chem. 121, 740-745 (2010)

[24] H. Kelebek, M. Jourdes, S. Selli, P-L. Teissedre, J. Sci. Food. Agric. 93, 2963-2972 (2013)

[25] L.F. Wessner, S.K. Kurtural, Am. J. Enol. Vitic. 64, 134-138 (2013) 
[26] S.K. Kurtural, L.F. Wessner, G. Dervishian, Hortscience 48, 576-583 (2013)

[27] N. Göktürk Baydar, Z. Babalık, F. Hallaç Türk, E.S. Çetin, J. of Agricultural Sciences 17, 67-76 (2011)

[28] R. Montealegre, R. Romero Peces, J.L. Chacón Vozmediano, J. Martinez Gascueña, E. Garcia Romero, J. of Food Composition and Analysis 19, 687-693 (2006)
[29] D. Rusjan, Z. Korosec-Koruza, Acta Chim. Slov. 54, 114-118 (2007)

[30] M.C. Benismail, M. Bennaouar, A. Elmribti, Int. Workshop on Advances in Grapevine and Wine Research, ISHS Acta Horticulturae 754. (2007)

[31] M.I.F. Fawzi, M.F.M. Shahin, E.A. Kandil, J. of Amer. Sci. 6, 187-194 (2010) 